\title{
Spatial Perspective on Regional Innovation System
}

\author{
Anna A. Mikhaylova \\ Immanuel Kant Baltic Federal University, Russian Federation \\ Email: tikhonova.1989@mail.ru
}

\section{Doi:10.5901/mjss.2015.v6n3s5p27}

\begin{abstract}
Article deals with the peculiarities in modeling the regional innovation system (RIS) as a form of territorial social system characterized by a specific set of properties of the systemic, innovative and spatio-positional nature. The author analyzes the existing models of RIS with an emphasis on the formation of generalized ideas about the composition, structure and relations of RIS with the external environment. The study suggests a unique approach to the modeling of RIS, which is defined as a localized cluster-network system that combines different types of actors being systematically involved in the innovation process embedded in the institutional environment of the region. The problem of spatial boundaries of RIS in the scale of national innovation and regional systems is considered.
\end{abstract}

Keywords: regional innovation system, national innovation system, region, structural model, composition model, innovation, cluster

\section{Introduction}

Fundamentals of the regional innovation system (RIS) concept were laid down in the 90s of the twentieth century (Cooke, 1992; Cooke \& Morgan, 1998; Maskell \& Malmberg, 1997) and received subsequent development in later works (see Asheim \& Isaksen, 2002; Isaksen, 2001; Doloreux \& Parto, 2004; Asheim \& Gertler, 2005). According to Cooke and colleagues (1998), RIS can be defined as a system that combines companies and organizations that are systematically involved in the process of interactive learning, embedded in the institutional environment of the region. It is generally accepted that the internal structure of RIS includes technical and economic (i.e. productive), as well as institutional components (Asheim \& Isaksen, 1997). This division is due to primordial notions of RIS as a set of regional clusters of firms (primarily industrial), surrounded by a supporting infrastructure, including education institutions, technology transfer agencies, business associations, financial institutions, research institutes, etc. (Asheim \& Isaksen, 2002). This approach provides scientists an opportunity to trace the development of the regional economy: starting from the creation of a regional cluster to its transformation into RIS via the processes of the formation of local innovative milieu, strengthening of formal inter-firm innovation cooperation, development of institutional infrastructure and increasing the numbers of knowledge suppliers. Regional cluster acts as an important prerequisite to the emergence of a full-fledged innovation system in the region, since it is being its nucleus (i.e. the core). However, despite the proximity of the cluster and RIS, RIS is generally more difficult territorial and social system. Therefore, using the existing cluster models is insufficient for the spatial modeling of innovative processes in the RIS. Rather, it requires the development of specialized models that take into account its specific features.

\section{Advantages of the RIS Approach to the Study of the Region}

The whole idea of the existence of regional innovation systems originated in the concept of a national innovation system (NIS), elaborated by K. Freeman, B.-A. Lundvall, and R. Nelson. In general terms, the NIS is understood as the network of institutions of a different kind that belong to both the private and public sectors whose activities and interactions provide for effective innovation processes at the national level (Surin \& Molchanova, 2008; Atoyan \& Kazakova, 2007; Selyaninov \& Frolova, 2012; Nelson, 1993). According to Lundvall (1992), NIS is a social dynamic system, which involves the circulation of innovation and new knowledge through a feedback mechanism between its elements as a result of social activity and interactive learning. The innovation process in such a system is described of the property of recursion.

At this point in the world of science, the question of subordination of NIS and RIS has no clear answer. There can be distinguished two main approaches to establish the relationship between the scale of national and regional innovation systems (Doloreux \& Parto, 2004). The first approach involves consideration of the region's innovation system as a 
subsystem of the NIS. The second approach is based on the concept of the region as an object whose boundaries are not strictly defined, which enables us to consider mega, macro, meso and micro-regions. In this context, both the country and its subject are regions: the differences are hidden in the scale of particular research.

The preferential use of RIS approach over NIS is justified in cases with a strong differentiation between regions within the country (especially in the case of federal states, where the powers of regional authorities are wider) or a strong regional identity that enables to isolate certain regions in the framework of research (e.g. Flanders and Wallonia in Belgium, the Basque Country in Spain, Scotland and Wales in UK, etc.). Cooke et al. (1997), while discussing the aspects of national innovation systems, note that the term "national", in addition to its traditional meaning of "state" may also be considered in the context of the nation as a united people, united by a common language, culture and territory. The absence of such unity creates the conditions for regional differences. A study of innovation systems at the mesolevel allows identifying those specific features that distinguish one region from another and determine its competitive advantage in a globalizing world. In this context, research approaches of RIS and NIS are characterized by significant differences (Korres, 2013), as the same aspects of innovation can take on quite different properties.

\section{Features of Modeling Regional Innovation Systems}

Scientific literature demonstrates no uniform principles for constructing the model of RIS, which is due to an empirical variety of phenomena as well as the differences in the research objectives. However, since the RIS is a system, a systemic approach should be used in its simulation, involving not only a description of its composition and internal structure, but also relations with the external environment. Analysis of 26 different models of innovation systems of the region, developed by Russian and foreign scientists, has enabled to develop a fundamental understanding of the RIS, which can be subsequently used as a basis of specialized models.

\subsection{Models on the composition of RIS}

From the standpoint of the research on RIS composition, of particular interest are the studies of Monastyrniy (2005), Fedulova and Pashuta (2005), Marakhovskiy (2009), Berezhnaya and Smirnova (2011), Chegodaeva (2012), Zadumkin (2008), Lapaev (2013), Sukhovey and Golova (2007), Shabaykova and Mezhetskaya (2011), Terebova and Gubanov (2009). Representation of the RIS structure in most of these models is realized through the description of a set of subsystems and their individual elements. The basic principle of separation into subsystems is functional, based on scientists' perceptions over the stages of the innovation process. The main subsystems being allocated are a) the production of new knowledge and ideas; b) commercialization and practical application of knowledge; c) support and dissemination of knowledge (Fedulova \& Pashuta, 2005; Sukhovey \& Golova, 2007; Marakhovskiy, 2009; Chegodaeva, 2012). It should be noted that the majority of authors using functional principle in decomposition of RIS into components are based on linear representations of the stages of the innovation process. However, the linear approach to innovation is largely simplified and often distorts the actual processes taking place in RIS. Thus, its use in modeling has certain limitations and must be justified for research purposes.

In addition to the functional principle of describing the structure of RIS, there is a principle of compositional unity, which is equally widespread. For example, Berezhnaya and Smirnova (2011) consider innovation system in the region as a set of scientific and educational, infrastructural, entrepreneurial and resource subsystems. A similar approach adheres Romanova (2013), further highlighting the innovation policy subsystem. Aralbaeva and Kuzaeva (2013) represent the composition of RIS by supportive, resource, organizational and functional subsystems. Comparative analysis of structure models of RIS and regional system demonstrates the close relationship and involvement of many RIS subsystems in to the subsystems of the regional system. For example, the resource subsystem of RIS combines natural, technical, technological, financial, labor, and informational resources, which can be compared with the industrial, financial, social, ecological and geographical subsystems of the regional system. The supportive subsystem of RIS - with political and legal subsystem of a region, entrepreneurial - with economic, etc. On this basis, we can conclude that RIS is a more ambitious (i.e. broad) system, rather than S\&T or an element of economic subsystem of the region, as it is sometimes considered.

In order to identify the views of the scientific community about the correlation of RIS and regional system, we carried out an interactive poll on a dedicated website researchgate.net, which is a platform that combines the world's leading scientists from different fields of knowledge. The survey involved ten experts from Argentina, Belgium, Colombia, Germany, Italy, Poland, Portugal, and the UK. Firstly, many experts had indicated the methodological problem in determining the boundaries of the region associated with differences in the scale of economic specialization, 
administrative structure and culture. Secondly, the majority of respondents expressed the view that the allocation of RIS in the regional system has no unique solution. The result is determined by the scope and objectives of the study. Since RIS can simultaneously perform the role of a surveillance tool and an object of study that exists within the boundaries of a particular region, its complex systemic structure is not determined. In other words, RIS is characterized by a polystructural feature. Allocation of certain elements within the structure of RIS relates to the specific features of the region on the one hand and their essential value for the study on the other. According to experts, RIS is primarily a system of links between actors and institutions concerned with the development of new technologies, expansion of innovative activity, and innovation support in the region. Strength and systematic nature of links within RIS varies between regions and determines their innovativeness. Researcher approach may draw conditional (i.e. nominal) boundaries while separating RIS from the rest of the world, but, as a rule, RIS is an open system, which is affected by a range of factors: economic, social, institutional, organizational and others. In this context, it is the remark of one of the experts that is of particular interest: due to certain reasons, not all administrative regions can incorporate RIS.

To summarize the foregoing, it is proposed to allocate broad and narrow approaches to the RIS concerning its subordination to the regional system. In broad terms, RIS can be equated to the region in which it is embedded. This is consistent with the non-linear representations of the innovation process, which examine the links between business and the research sector, as well as the government, non-profit institutions, and civil society. In a narrow sense, RIS is a subsystem of the regional system, reflecting its most striking properties within a given research program.

Regardless of the chosen approach to the construction of RIS model, each of the subsystems allocated in its part can be divided into separate elements. The research of Monastyrniy (2005), Chistyakova (2007), Lapaev (2013), Egorova and Avilova (2007), Slepnava (2014) reflect the groupings of the RIS elements according to their role in the innovation process: a) "principal activity"; b) "supportive of the principal activity"; c) "control / regulation" (by regional authorities and administration). Having generalized the existing approaches to elemental composition in the logic of a broad approach to RIS, the following model of regional innovation system is proposed (see Table 1).

Table 1. Composition model of Regional Innovation System

\begin{tabular}{lll}
\hline \multicolumn{1}{c}{ System } & \multicolumn{1}{c}{ Subsystem } & \multicolumn{1}{c}{ Elements } \\
\hline $\begin{array}{l}\text { Regional } \\
\text { Innovation } \\
\text { System }\end{array}$ & TNCs; large, small and medium enterprises in the manufacturing industry, \\
& agriculture, fisheries and fish farming, mining, construction, manufacturing \\
& and distribution of electricity, gas and water (i.e. utilities sector), wholesale \\
& and retail trade, transport and communication, hotel and restaurant business; \\
& small innovative companies; organizations engaged in financial activities (e.g. \\
& insurance companies, financial and credit institutions, leasing and investment \\
& firms, financial funds, sponsorship funds, etc.); companies that provide a \\
& variety of services (e.g. consulting, auditing, accounting, certification, \\
& licensing, patenting, examination, design, etc.) and others. \\
\cline { 2 - 3 } & Organizations involved in basic and applied research and development (e.g. \\
& $\begin{array}{l}\text { R\&D organizations, higher education research organizations, experimental } \\
\text { Scientific and Technological }\end{array}$ \\
& design, engineering design, design and technology organizations), industrial \\
& parks, business incubators, innovation centers, scientific and technological \\
& centers, etc. \\
\hline Social & Organizations in the field of education (e.g. university, college, NGOs, training \\
& centers, etc.), health and social services, households, social institutions, a \\
& pool of highly qualified specialists, etc. \\
\hline Political and Legal & Regional authorities and administration, political organizations and other \\
& political institutions, legal and regulatory framework, etc. \\
\hline
\end{tabular}

\subsection{Model of the RIS structure}

Structural model of RIS should reflect the interrelation of its elements. In this respect, of particular interest are the publications of Uotila and colleagues (2006), Cooke and Piccaluga (2004), Monastyrniy and Chistyakova (2007), Prokopenko (2009), Chernova (2010), Shaybakova and Mezhetskaya (2011), Terebova and Gubanov (2009), Shaposhnikova (2008), Zhilnikov (2013) and a number of others. Most of the basic models examine the relationships between the subsystems of RIS being placed in a contextual environment by simply listing the elements of the system. Specification (i.e. detailing) of such models by defining the linkages between the individual elements is often complicated 
because of the high degree of uncertainty. The main mechanism for ensuring cooperation in RIS is the innovation process (Egorova, 2008; Egorova \& Avilova, 2007; Shaposhnikova, 2008). In the real RIS, the links being formed between the elements are heterogeneous. They differ in the nature of construction (vertical and horizontal or literal), the degree of rigidity (strong and weak), duration (short, medium, long-term) (Hadjimanolis, 1999). This is partly reflected in the model of Uotila et al. (2006).

Vertical linkages are formed in the value chains, linking the company with its suppliers and customers. Most of these links are formal and legislated in the form of contracts, purchase and sale agreements, etc., either one-off or longterm. Value chain interaction over a long period enables to equalize the level of technical and technological development of enterprises and helps the gradual merging of their processes. The consequence is a growing interdependence and complementarity of legally independent firms. Horizontal linkages are more intermediate and generally are latent information channels of tacit knowledge sharing. An example of such bonds are the simulation processes of competing firms, the short-term linkages with scientific and educational institutions, and public authorities, etc. Achieving the localization of a critical mass of cooperating and competing companies and their transform into a single system (e.g., industrial cluster of a satellite or stellar structure; see Boja, 2011), we can talk about the formation of horizontal links of a certain sustainability level.

Despite the latest arguments on the increasing importance of horizontal inter-organizational interactions, studies of a number of scholars demonstrate the not diminishing role of vertical ties, including with regard to the generation, commercialization and diffusion of innovations (Cooke et al., 2000; Doloreux, 2004; Nieto \& Santamaria, 2007; Whitley, 2002). Scientists note that the cooperation within the hierarchical structure from suppliers to consumers is especially important for small and medium-sized companies, the limited internal resources of which does not allow them to implement large-scale innovation projects on their own.

Configuration features of horizontal and vertical linkages between regional actors in RIS cause diverse forms of economic cooperation: clusters, networks, value chains, etc. Each of these forms has its own specific properties and can be the subject of a separate study.

\subsection{Communication of RIS with the external environment}

Traditionally RIS is considered as an open system that interacts with the rest of the material world. Simplified representation of the system of "RIS - external environment" relations can be obtained from the analysis of models built on the "black box" principle (Chernova, 2010; Chistyakova, 2007; Lapaev, 2013; Slepneva, 2014). Different kinds of resources are considered as input flows in these models: informational, material, financial, human, technical and technological. After entering RIS, these inputs transform under the influence of a combination of factors: economic, social, cultural, technological, institutional. These factors can be positive, while acting as a stimulus for the development of RIS, and negative, acting as barriers (Mikhaylova \& Mikhaylov, 2015). At the exit of the system the new resource flows are formed, including innovative products and services, highly qualified staff, educational technology, scientific knowledge, organizational and managerial experience, patents and licenses, inventions, developments, know-how, new technologies, legislative initiatives, etc. Another significant influence on the innovation processes in the RIS has globalization, and as a consequence, a growing international and interregional competition.

\section{Spatio-network Model of RIS}

RIS, as an object of study, is a localized cluster-network system that combines different kinds of actors being systematically involved in the innovation process and embedded in the institutional environment of the region. We have identified systemic, innovative and spatio-positional properties of RIS (Figure 1). 


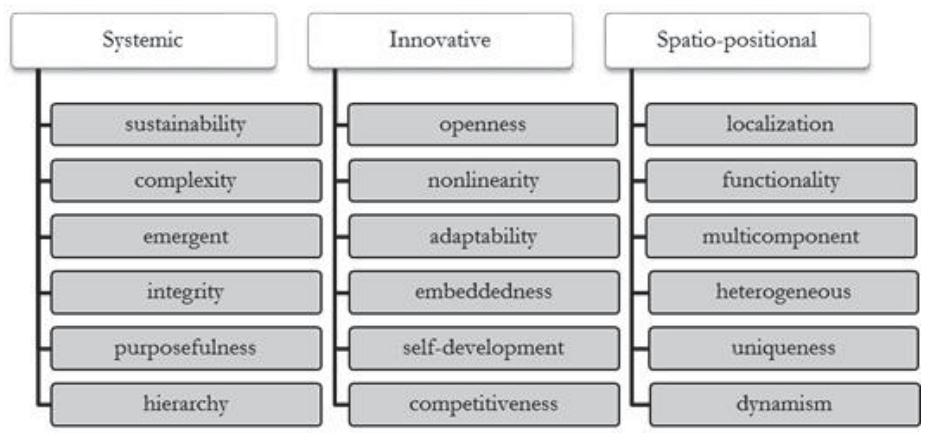

Figure 1. Properties of the regional innovation system

The first group of properties were classified as the reflection of the systemic nature of RIS:

sustainability - the ability of RIS to self-development in the long run;

complexity - describes the structure of the RIS, including a plurality of interacting elements, each of which can also be considered as a complex system;

emergence (or emergent) - irreducibility of RIS properties to the simple sum of the properties of its elements (as a consequence, the possibility of achieving synergy in RIS);

integrity - defined by the interconnectedness of the elements of RIS, i.e. change in the parameters (properties, structure, functions, etc.) of one or more elements of RIS affects the other elements and the system as a whole;

purposefulness - the property of all the elements of RIS to function together as to achieve a common vision, a common goal; its structure.

hierarchy - reflects the multi-level nature of RIS, i.e. defines the possibility to allocate several hierarchical levels in

The second group includes properties that are acquired by the system under the influence of innovation components:

openness - characterizes the presence of network links of the RIS with the external environment, its involvement in various communication channels (especially in the exchange flows of new knowledge and innovation);

nonlinearity - assumes the network character of interactions between the elements of the RIS, a combination of vertical and horizontal linkages, the presence of backward links (this property is largely due to the occurrence of innovation processes in the RIS, which are not linear in themselves);

adaptability - the ability to change the characteristics of RIS in the process of adaptation to changing external and / or internal conditions by generating new combinations of existing resources and ensure the provision of necessary external resources;

embeddedness - property of RIS reflecting that innovation processes are being rooted in the environment in which they occur;

self-development - the ability of RIS to a qualitative self-improvement in the current macro environment mainly via the domestic development sources (primary - the intellectual capital);

competitiveness - a property that characterizes the ability of RIS to compete at various hierarchical levels through the implementation of its internal (incl. hidden) capacity in order to ensure the rapid socio-economic development of the region.

Identification of the third group of properties is due to the perception of RIS as territorial socio-economic system, which takes a definite place in the geospace:

localization - belonging to a certain area;

functionality - characterizes the emergence of a set of feature or properties due to the peculiarities of the geographical location of RIS and its interaction with the environment;

multicomponent - reflects the structural complexity of RIS that involves interaction of various subsystems of the region (economic, social, political, scientific, technical, environmental);

heterogeneity - characterizes the spatial differences in the level of innovative development, resulting in the center and periphery structure of the RIS;

uniqueness - due to the presence of particular specific properties intrinsic to a given RIS; 
dynamism - continuous variability of RIS and the contextual environment over time.

Generalization of scientific ideas about the composition, structure of RIS and its interactions with the external environment resulted in to the development a fundamental spatio-network model of the innovation system of the region (Figure 2).

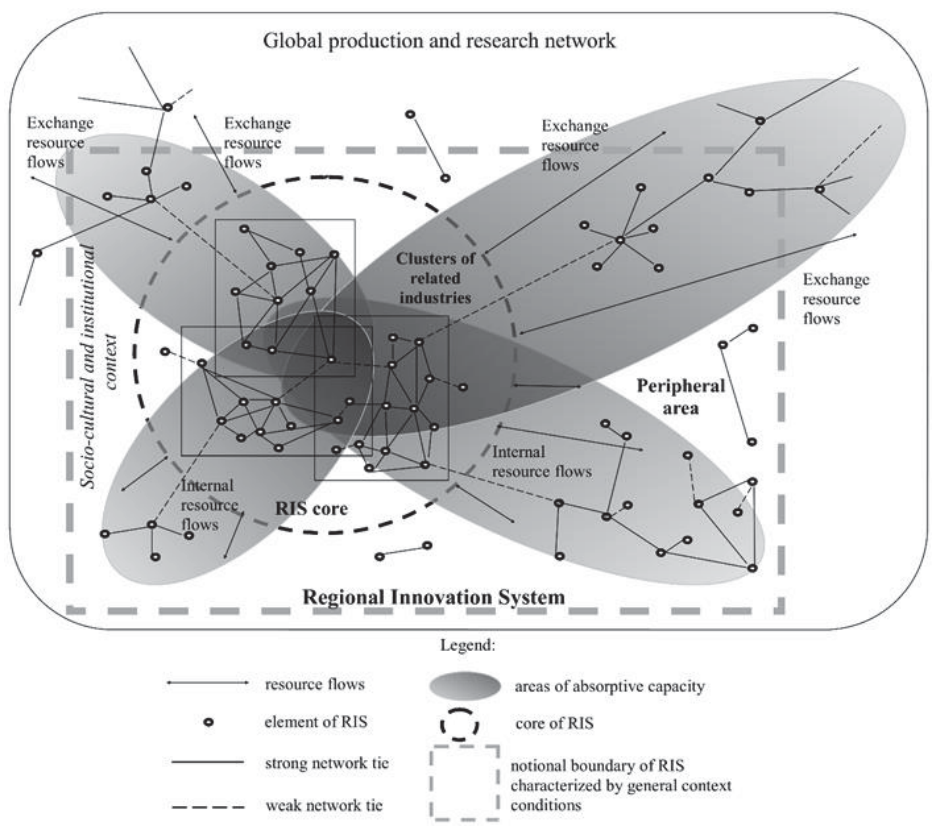

Figure 2. Schematic model of regional innovation system

The proposed model is based on a broad approach to RIS within the meaning of the regional system that takes into account the diversity of the actors directly and indirectly involved in the innovation process. Regional system is considered in the administrative-territorial boundaries of the region. Territorial heterogeneity of innovative development is reflected in the functional type of the region, which is characterized by the presence of the core and the associated peripheral area. The core of the RIS is formed by clusters of interdependent and complementary industries. The boundaries of the clusters are indicative and may be interpreted by allocating long-term sustainable aggregations of strong vertical and horizontal inter-sectoral linkages of the regional network. Over time, the partial or complete reconfiguration of links between industries with the core of the RIS is possible, the consequence of which is the formation of new value chains, clusters, and even industries (Ketels \& Protsiv, 2014).

Between the core and peripheral zones the resource flows are formed, by which there is a movement of various resources within the RIS. As a rule, the core of the RIS, being the main attractor, attracts the main share of human, financial, investment and intellectual resources of the region. The diffusion of knowledge and innovation between the core and the periphery occurs mainly through a network of weak ties, which form areas of absorptive capacity. There can be distinguished two types of areas: the potential areas, associated with the assimilation of knowledge, and realized areas, related to the conversion and use of knowledge (Uotila et al., 2006). Currently, the economic geography attempts to determine the conditional maximum distribution boundaries of the absorptive capacity (Mikhaylov, 2014). There is a significant impact of transport and ICT infrastructure development level on the geographical areas of absorption, both intra-regional and those linking the region with the rest of the world.

\section{Conclusion}

RIS is a complex territorial social system, the development dynamics of which is influenced by a large number of internal and external factors of different nature (economic, social, institutional, foreign policy, organizational, cultural, historical, etc.). The results of empirical studies carried out by scientists at a variety of regions in Sweden, Norway, USA, Canada, 
China, Russia, etc., is the understanding that there is a whole variety of different types of RIS, which makes it impossible to develop universal solutions and models. This largely explains the diversity of RIS models being represented in the scientific literature.

However, despite the specificity of the RIS of specific regions, the development of the RIS model should consider the following. Firstly, before modeling the RIS, one should define the boundaries of the region under study (it was also indicated by the experts involved in the survey). In this case, the concept of borders incorporates not only the geographical content, but also political and legal, as well as cultural and historical. The concept of region is also rather wide and diverse. Different countries have their own administrative-territorial divisions. Therefore, the issue of spatial boundaries is an important methodological aspect of modeling. Secondly, as RIS relates to the class of complex systems, its model will always be a simplified reflection of reality. In constructing the model the most significant factors should be considered, the choice of which is conditioned by research objectives. Third, the construction of a RIS model requires consideration of its objective properties that can be divided into three groups: systemic, innovative and spatio-positional. Fourth, a great influence on the results of RIS modeling have the representations on the breadth and embeddedness of innovative processes taking place in the region, the degree of subordination of RIS in the region and NIS of the country, the level of integration of the region into the international innovation space.

Analysis of 26 different models of RIS conducted in the framework of this study has provided an opportunity to summarize the scientific ideas (predominantly of Russian scholars) on the composition and structure of the RIS and especially its interactions with the external environment. Were identified a broad and narrow approaches towards understanding and simulation of RIS. In the first approach, RIS is considered within the boundaries of the regional system, in the second - as one of its subsystems. Following the logic of the broad approach, author proposed the spationetwork model of RIS, which takes into account the territorial factor, unlike most other models of RIS. Using this model will enable to study the composition of the RIS of a specific region, the various types of relationships between its elements, as well as their relative spatial location, allocate the core, peripherals and the areas of absorptive capacity.

\section{References}

Aralbaeva, F. Z., \& Kuzaeva, T. V. (2011). Structure of an innovation subsystem in the regional socio-economic system. Vestnik OSU, 13(132), 30-32. [in Russian]

Asheim, B. T., \& Gertler, M. S. (2005). The Geography of Innovation. Regional Innovation Systems. In J. Fagerberg, D. C. Mowery, \& R. R. Nelson (eds.) The Oxford Handbook of Innovation (pp. 291-317). Oxford University Press.

Asheim, B. T., \& Isaksen, A. (1997). Location, agglomeration and innovation: towards regional innovation systems in Norway? European Planning Studies, 5 (3), 299-330.

Asheim, B. T., \& Isaksen, A. (2002). Regional Innovations Systems: The Integration of Local 'Sticky' and Global 'Ubiquitious Knowledge'. The Journal of Technological Transfer, 27, 77-88.

Atoyan, V. R., \& Kazakova, N. V. (2007). Some approaches to the analysis of the development of innovative systems in a globalizing world. Innovations, 3, 27-34. [in Russian]

Berezhnaya, I. V., \& Smirnova, E. A. (2011). Structural model of regional innovation system. Economics and Management, 2, 54-59. [in Russian]

Boja, C. (2011). Clusters Models, Factors and Characteristics. International Journal of Economic Practices and Theories, 1 (1), 34-43

Chegodaeva, A. N. (2012). The development of regional innovation systems. Synopsis of dissertation for the degree of candidate of economic sciences: Sergei Witte Moscow University, Moscow. [in Russian]

Chernova, O. A. (2010). Subject-object definition of a regional innovation system. Bulletin of Tomsk State University, 4 (12), 149-158. [in Russian]

Chistyakova, N. O. (2007). Regional innovation system: model, structure, specificity. Innovations, 4, 55-58. [in Russian]

Cooke, P. (1992). Regional innovation systems: competitive regulation in the new Europe. Geoforum, 23, 365-382.

Cooke, P., \& Morgan, K. (1998). The associational economy. Firms, regions, and innovation. Oxford: Oxford University Press.

Cooke, P., \& Piccaluga, A. (2004). Regional Economies As Knowledge Laboratories. Cheltenham: Edward Elgar.

Cooke, P., Boekholt, P., \& Tǒdtling, F. (2000). The Governance of Innovation in Europe. London: Pinter.

Cooke, P., Uranga, M. G., \& Etxebarria, G. (1997). Regional innovation systems: Institutional and organizational dimensions. Research Policy, 26, 475-491.

Cooke, P., Uranga, M. G., \& Etxebarria, G. (1998). Regional systems of innovation: an evolutionary perspective. Environment and Planning A, 30, 1563-1584.

Doloreux, D. (2004). Regional networks of small and medium sized enterprises: evidence from the metropolitan area of Ottawa in Canada. European Planning Studies, 12 (2), 173-189.

Doloreux, D., \& Parto, S. (2004). Regional Innovation Systems: A Critical Synthesis. Discussion Papers, 17. UNU-INTECH.

Egorova, M. V. (2008). Concept of regulation of the formation and functioning of the regional innovation system. Innovations, 7 (117), $91-$ 93. [in Russian] 
Egorova, M. V., \& Avilova, V. V. (2007). Model of regional innovation systems: theoretical and methodological aspects. Innovations, 6 (104), 66-69. [in Russian]

Fedulova, L. I., \& Pashuta, M. T. (2005). Development of the national innovation system of Ukraine. Ukraine's economy, 4 (521), $35-47$. [in Russian]

Hadjimanolis, A. (1999). Barriers to innovation for SMEs in a small less developed country (Cyprus). Technovation, 19 (9), 561-570.

Isaksen, A. (2001). Building regional innovation systems: is endogenous industrial development possible in the global economy? Canadian Journal of Regional Science, 24 (1), 101- 120.

Ketels, Ch., \& Protsiv, S. (2014). Methodology and Findings Report for a Cluster Mapping of Related Sectors. European Cluster Observatory. [Online] Available: http://ec.europa.eu/enterprise/initiatives/cluster/observatory/d1.2-cluster-mappingreport.pdf (December 14, 2014).

Korres, G. M. (2013). The European national and regional systems of innovation. In E. G. Carayannis \& G. M. Korres (eds.) The Innovation Union in Europe: A Socio-Economic Perspective on EU Integration (pp. 85-98). Edward Elgar Publishing Limited.

Lapaev, S. P. (2013). Management of formation of the regional innovation system. Doc. thesis: Orenburg State University, Orenburg. [in Russian]

Lundvall, B- $\AA$. (1992). National systems of innovation: towards a theory of innovation and interactive learning. London: Pinter.

Marakhovskiy, A. A. (2009). Some features of the relationship of innovation systems in Ukraine. Business INFORM, 4 (1), 76 - 80. [in Russian]

Maskell, P., \& Malmberg, A. (1997). Towards an explanation of regional specialization and industry agglomeration. European Planning Studies, 5 (1), 25-41.

Mikhaylov, A. S. (2014). International cluster as a form of territorial organization of the economy of the Baltic region in the context of globalization. PhD thesis: IKBFU, Kaliningrad. [in Russian]

Mikhaylova, A. A., \& Mikhaylov, A. S. (2015). Antecedents and Barriers to the Formation of Regional Innovation System: Case Study of the Kaliningrad Region. Modern Applied Science, 9 (2), 178-187.

Monastyrniy, E. A. (2005). Structural model of the innovation system. Innovations, 8, 49-54. [in Russian]

Monastyrniy, E. A., \& Chistyakov, N. O. (2007). Structural-functional model of subsystem 'Infrastructure' in the regional innovation system. Innovations, 6 (104), 58-65. [in Russian]

Nelson, R. R. (1993). National Innovation Systems: A comparative analysis. New York, Oxford: Oxford University Press.

Nieto, M. J., \& Santamaria, L. (2007). The importance of diverse collaborative networks for the novelty of product innovation. Technovation, 27(6-7), 367-377.

Prokopenko, E. S. (2009). Conditions and factors of the effective operation of the regional innovation system. PhD thesis: Institute of Management, Business and Law, Rostov-on-Don. [in Russian]

Romanova, M. V. (2013). Formation and development of regional innovation system. PhD thesis: Russian University of Cooperation, Moscow. [in Russian]

Selyaninov, A. V., \& Frolova, N. V. (2012). The practical application of the principles of efficiency and sustainability in the management of national and regional innovation systems. Innovation Management, 4, 81-93. [in Russian]

Shaposhnikova, S. V. (2008). Management of different types of innovation systems. InVestRegion, 4, 27-31. [in Russian]

Shaybakova, L. F., \& Mezhetskaya, A. L. (2011). Formation of regional innovation systems in Russia. News USUE, 2 (34), 92-98. [in Russian]

Slepneva, Y. V. (2014). Mechanism to ensure intellectual development of regional innovation systems. Synopsis of dissertation for the degree of candidate of economic sciences: East Siberian State University of Technology and Management, Ulan-Ude. [in Russian]

Sukhovey, A. F., \& Golova, I. M. (2007). Problems of innovative activities in the context of the formation of regional innovation systems. The region's economy, 3, 111-122. [in Russian]

Surin, A. V., \& Molchanova, O. P. (2008). Innovation Management: Textbook. Moscow: INFRA-M. [in Russian]

Terebova, S. V., \& Gubanov, E. S. (2009). Activation of the innovation process in the region: monograph. Vologda: VSCC CEMI. [in Russian]

Uotila, T., Harmaakorpi, V., \& Melkas, H. (2006). A method for assessing absorptive capacity of a regional innovation system. Fennia, 1 (184), 49- 58.

Whitley, R. (2002). Developing innovative competences: the role of institutional frameworks. Industrial and Corporate Change, 11(3), 497-528.

Zadumkin, K. A., \& Kondakov, I. A. (2008). Regional Innovation System: theory and practice of formation. Vologda: Vologda Research Coordination Center CEMI. [in Russian]

Zhilnikov, A. Y. (2013). Model for predicting the trajectory of regional development. Belgorod State University, 15-1 (158), 98-105. [in Russian] 